\title{
Research Article \\ Critical Point Theorems for Nonlinear Dynamical Systems and Their Applications
}

\author{
Wei-Shih Du \\ Department of Mathematics, National Kaohsiung Normal University, Kaohsiung 802, Taiwan \\ Correspondence should be addressed to Wei-Shih Du, wsdu@nknucc.nknu.edu.tw \\ Received 9 February 2010; Accepted 18 April 2010 \\ Academic Editor: Lai Jiu Lin
}

Copyright $(2010$ Wei-Shih Du. This is an open access article distributed under the Creative Commons Attribution License, which permits unrestricted use, distribution, and reproduction in any medium, provided the original work is properly cited.

We present some new critical point theorems for nonlinear dynamical systems which are generalizations of Dancš-Hegedüs-Medvegyev's principle in uniform spaces and metric spaces by applying an abstract maximal element principle established by Lin and Du. We establish some generalizations of Ekeland's variational principle, Caristi's common fixed point theorem for multivalued maps, Takahashi's nonconvex minimization theorem, and common fuzzy fixed point theorem for $\tau$-functions. Some applications to the existence theorems of nonconvex versions of variational inclusion and disclusion problems in metric spaces are also given.

\section{Introduction}

In 1983, Dancš et al. [1] proved the following existence theorem of critical point (or stationary point or strict fixed point) for a nonlinear dynamical system.

\section{Dancš-Hegedüs-Medvegyev's Principle [1]}

Let $(X, d)$ be a complete metric space. Let $\Gamma: X \rightarrow 2^{X}$ be a multivalued map with nonempty values. Suppose that the following conditions are satisfied:

(i) for each $x \in X$, we have $x \in \Gamma(x)$ and $\Gamma(x)$ is closed,

(ii) $x, y \in X$ with $y \in \Gamma(x)$ implies that $\Gamma(y) \subseteq \Gamma(x)$,

(iii) for each $n \in \mathbb{N}$ and each $x_{n+1} \in \Gamma\left(x_{n}\right)$, we have $\lim _{n \rightarrow \infty} d\left(x_{n}, x_{n+1}\right)=0$.

Then there exists $v \in X$ such that $\Gamma(v)=\{v\}$.

The famous Dancš-Hegedüs-Medvegyev's Principle is an important tool in various fields of applied mathematical analysis and nonlinear analysis. A number of generalizations 
of these results have been investigated by several authors; for example, see $[2,3]$ and references therein.

In 1963, Bishop and Phelps [4] proved a fundamental theorem concerning the density of the set of support points of a closed convex subset of a Banach space by using a maximal element principle in certain partially ordered complete subsets of a normed linear space. Later, the famous Brézis-Browder's maximal element principle [5] was established and applied to deal with nonlinear problems. Many generalizations in various different directions of maximal element principle have been studied in the past; for example, see $[2,3,6-10]$ and references therein. However, few literatures are concerned with how to define a sufficient condition for a nondecreasing sequence on a quasiordered set to have an upper bound. Recently, Du [7] and Lin and Du [3] defined the concepts of sizing-up function and $\mu$ bounded quasiordered set (see Definitions 1.1 and 1.3 below) to describe a rational condition for a nondecreasing sequence on a quasiordered set to have an upper bound.

Definition 1.1 (see $[3,7]$ ). Let $X$ be a nonempty set. A function $\mu: 2^{X} \rightarrow[0, \infty]$ defined on the power set $2^{X}$ of $X$ is called sizing-up if it satisfies the following properties:

$$
\begin{aligned}
& (\mu 1) \mu(\emptyset)=0, \\
& (\mu 2) \mu(A) \leq \mu(B) \text { if } A \subseteq B .
\end{aligned}
$$

Definition 1.2 (see $[3,7]$ ). Let $X$ be a nonempty set and $\mu: 2^{X} \rightarrow[0, \infty]$ a sizing-up function. A multivalued map $T: X \rightarrow 2^{X}$ with nonempty values is said to be of type $(\mu)$ if, for each $x \in X$ and $\varepsilon>0$, there exists a $y=y(x, \varepsilon) \in T(x)$ such that $\mu(T(y)) \leq \varepsilon$.

Definition 1.3 (see $[3,7]$ ). A quasiordered set $(X, \lesssim)$ with a sizing-up function $\mu: 2^{X} \rightarrow$ $[0, \infty]$, in short $(X, \lesssim, \mu)$, is said to be $\mu$-bounded if every nondecreasing sequence $x_{1} \lesssim x_{2} \lesssim$ $\cdots \lesssim x_{n} \lesssim x_{n+1} \lesssim \cdots$ in $X$ satisfying

$$
\lim _{n \rightarrow \infty} \mu\left(\left\{x_{n}, x_{n+1}, \ldots\right\}\right)=0
$$

has an upper bound.

In [7] (see also [3]), Lin and Du established the following abstract maximal element principle in a $\mu$-bounded quasiordered set with a sizing-up function $\mu$.

Theorem LD [see [3, 7]]

Let $(X, \lesssim, \mu)$ be a $\mu$-bounded quasiordered set with a sizing-up function $\mu: 2^{X} \rightarrow[0, \infty]$. For each $x \in X$, let $S: X \rightarrow 2^{X}$ be defined by $S(x)=\{y \in X: x \lesssim y\}$. If $S$ is of type $(\mu)$, then, for each $x_{0} \in X$, there exists a nondecreasing sequence $x_{0} \lesssim x_{1} \lesssim x_{2} \lesssim \cdots$ in $X$ and $v \in X$ such that

(i) $v$ is an upper bound of $\left\{x_{n}\right\}_{n=0}^{\infty}$,

(ii) $S(v) \subseteq \bigcap_{n=0}^{\infty} S\left(x_{n}\right)$,

(iii) $\mu\left(\bigcap_{n=0}^{\infty} S\left(x_{n}\right)\right)=\mu(S(v))=0$.

It is well known that Ekeland's variational principle is equivalent to Caristi's fixed point theorem, to Takahashi's nonconvex minimization theorem, to the drop theorem, and 
to the petal theoerm. Many generalizations in various different directions of these results in metric (or quasimetric) spaces and more general in topological vector spaces have been investigated by several authors in the past; for detail, one can refer to [2, 3, 7-9, 11-23]. By applying Theorem LD, Du [7] gave a generalized Brézis-Browder principle, system (vectorial) versions of Ekeland's variational principle and maximal element principle and a vectorial version of Takahashi's nonconvex minimization theorem. Moreover, the author investigated the equivalence between scalar versions and vectorial versions of these results. For more detail, one can see [7].

The paper is divided into four sections. In Section 3, we establish some new critical point theorems for nonlinear dynamical systems which are generalizations of Dancš, Hegedüs and Medvegyev's principles in uniform spaces and metric spaces by applying an abstract maximal element principle established by Lin and Du. We also give some generalizations of Ekeland's variational principle, Caristi's common fixed point theorem for multivalued maps, Takahashi's nonconvex minimization theorem, and common fuzzy fixed point theorem for $\tau$-functions. Some existence theorems of nonconvex versions of variational inclusion and disclusion problems in metric spaces are also given in Section 4. Our techniques and some results are quite original in the literatures.

\section{Preliminaries}

Let us begin with some basic definitions and notation that will be needed in this paper. Let $X$ be a nonempty set. A fuzzy set in $X$ is a function of $X$ into $[0,1]$. Let $\mathcal{F}(X)$ be the family of all fuzzy sets in $X$. A fuzzy map on $X$ is a map from $X$ into $\mathcal{F}(X)$. This enables us to regard each fuzzy map as a two-variable function of $X \times X$ into $[0,1]$. Let $F$ be a fuzzy map on $X$. An element $x$ of $X$ is said to be a fuzzy fixed point of $F$ if $F(x, x)=1$ (see, e.g., $[11,12,16,24-26]$ ). Let $\Gamma: X \rightarrow 2^{X}$ be a multivalued map. A point $x \in X$ is called a critical point (or stationary point or strict fixed point) $[1,3,8,27-29]$ of $\Gamma$ if $\Gamma(v)=\{v\}$.

Let " $\lesssim$ " be a quasiorder (preorder or pseudoorder, that is, a reflexive and transitive relation) on $X$. Then $(X, \lesssim)$ is called a quasiordered set. In a quasiordered set $(X, \lesssim)$, recall that an element $v$ in $X$ is called a maximal element of $X$ if there is no element $x$ of $X$, different from $v$, such that $v \lesssim x$. Denote by $\mathbb{R}$ and $\mathbb{N}$ the set of real numbers and the set of positive integers, respectively. A sequence $\left\{x_{n}\right\}_{n \in \mathbb{N}}$ in $X$ is called nondecreasing (resp., nonincreasing) if $x_{n} \lesssim x_{n+1}$ (resp., $x_{n+1} \lesssim x_{n}$ ) for each $n \in \mathbb{N}$.

Let $X$ be a nonempty set and $U, V$ any subsets of $X \times X$. Denote by

$$
\begin{gathered}
\Delta=\{(x, x): x \in X\} \quad \text { the diagonal of } X \times X, \\
U[x]=\{y \in X:(x, y) \in U\} \quad \text { the entourage of } x \in X, \\
U^{-1}=\{(x, y) \in X \times X:(y, x) \in U\}, \\
U \circ V=\{(x, y) \in X \times X:(z, y) \in U,(x, z) \in V \text { for some } z \in X\} .
\end{gathered}
$$

Recall that a uniform space $(X, \mathcal{U})$ is a nonempty set $X$ endowed of a uniformity $\mathcal{U}$, with the latter being a family of subsets of $X \times X$ and satisfying the following conditions:

(u1) $\Delta \subseteq V$ for any $V \in \mathcal{u}$,

(u2) If $V_{1}, V_{2} \in \mathcal{U}$, then there exists $W \in \mathcal{U}$ such that $W \subset V_{1} \cap V_{2}$, 
(u3) If $V \in \mathcal{U}$, then there exists $W \in \mathcal{U}$ such that $W \circ W^{-1} \subset V$,

(u4) If $V \in \mathcal{U}$ and $V \subset W \subset X \times X$, then $W \in \mathcal{U}$.

Two points $x$ and $y$ of $X$ are said to be $V$-close whenever $(x, y) \in V$ and $(y, x) \in V$. A sequence $\left\{x_{n}\right\}_{n \in \mathbb{N}}$ in $X$ is called a Cauchy sequence for $\mathfrak{U}((\mathcal{U})$-Cauchy sequence, for short) if, for any $V \in \mathcal{U}$, there exists $\ell \in \mathbb{N}$ such that $x_{n}$ and $x_{m}$ are $V$-close for $n, m \geq \ell$. A nonempty subset $C$ of $X$ is said to be sequentially $(\mathcal{U})$-complete if every $(\mathfrak{U})$-Cauchy sequence in $C$ converges. A uniformity $\mathcal{U}$ defines a unique topology $\tau(\mathcal{U})$ on $X$. A uniform space $(X, \mathcal{U})$ is said to be Hausdorff if and only if the intersection of all the $V \in \mathcal{U}$ reduces to the diagonal $\Delta$ of $X$, that is, if $(x, y) \in V$ for all $V \in \mathcal{U}$ implies that $x=y$. This guarantees the uniqueness of limits of sequences.

Let $(X, d)$ be a metric space. A real-valued function $f: X \rightarrow(-\infty, \infty]$ is said to be proper if $f \not \equiv \infty$. Recall that a function $p: X \times X \rightarrow[0, \infty)$ is called a $\tau$-function $[9,18]$, if the following conditions hold:

( $\tau 1) p(x, z) \leq p(x, y)+p(y, z)$ for all $x, y, z \in X$,

$(\tau 2)$ if $x \in X$ and $\left\{y_{n}\right\}$ in $X$ with $\lim _{n \rightarrow \infty} y_{n}=y$ such that $p\left(x, y_{n}\right) \leq M$ for some $M=M(x)>0$, then $p(x, y) \leq M$,

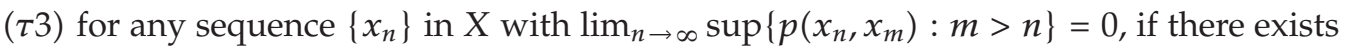
a sequence $\left\{y_{n}\right\}$ in $X$ such that $\lim _{n \rightarrow \infty} p\left(x_{n}, y_{n}\right)=0$, then $\lim _{n \rightarrow \infty} d\left(x_{n}, y_{n}\right)=0$,

( $\tau 4)$ for $x, y, z \in X, p(x, y)=0$ and $p(x, z)=0$ imply that $y=z$.

It is known that any $w$-distance $[15,18,19,21,22,30,31]$ is a $\tau$-function; see [18, Remark 2.1].

The following result is crucial in this paper.

Lemma 2.1. Let $(X, d)$ be a metric space and let $p: X \times X \rightarrow[0, \infty)$ be a function. Assume that $p$ satisfies condition ( $\tau 3)$. If a sequence $\left\{x_{n}\right\}$ in $X$ with $\lim _{n \rightarrow \infty} \sup \left\{p\left(x_{n}, x_{m}\right): m>n\right\}=0$, then $\left\{x_{n}\right\}$ is a Cauchy sequence in $X$.

Proof. Let $\left\{x_{n}\right\}$ in $X$ with $\lim _{n \rightarrow \infty} \sup \left\{p\left(x_{n}, x_{m}\right): m>n\right\}=0$. We claim that $\left\{x_{n}\right\}$ is a Cauchy sequence. For each $n \in \mathbb{N}$, let $\epsilon_{n}=\sup _{i, j>n} d\left(x_{i}, x_{j}\right)$. Then $\left\{\epsilon_{n}\right\}$ is nonincreasing and so $\lim _{n \rightarrow \infty} \epsilon_{n}:=\epsilon_{\infty}$ exists. If $\epsilon_{\infty}>0$, then there exist sequences $\left\{i_{n}\right\}$ and $\left\{j_{n}\right\}$ with $i_{n}<j_{n}$ such that $d\left(x_{i_{n}}, x_{j_{n}}\right)>(1 / 2) \epsilon_{\infty}$ for $n \in \mathbb{N}$. On the other hand, since $\lim _{n \rightarrow \infty} p\left(x_{i_{n}}, x_{j_{n}}\right)=0$, by $(\tau 3)$, we have $\lim _{n \rightarrow \infty} d\left(x_{i_{n}}, x_{j_{n}}\right)=0$, a contradiction. Therefore $\epsilon_{\infty}=0$ which shows that $\left\{x_{n}\right\}$ is a Cauchy sequence in $X$.

Remark 2.2. Notice that the function $p$ was assumed a $\tau$-function in [18, Lemma 2.1] and the proof of [18, Lemma 2.1] was incomplete since only $\lim _{n \rightarrow \infty} d\left(x_{n}, x_{n+1}\right)=0$ was demonstrated if any sequence $\left\{x_{n}\right\}$ in $X$ satisfied $\lim _{n \rightarrow \infty} \sup \left\{p\left(x_{n}, x_{m}\right): m>n\right\}=0$.

\section{New Critical Point Theorems in Uniform Spaces and Metric Spaces}

In this section, we will establish some new critical point theorems for nonlinear dynamical systems which are generalizations of Dancš-Hegedüs-Medvegyev's principle with common fuzzy fixed point in uniform spaces and metric spaces. 
Theorem 3.1. Let $X$ be a nonempty set, and let $\psi: X \times X \rightarrow(-\infty, \infty]$ and $\tau: X \rightarrow[-\infty, \infty)$ be functions. Let $\Phi$ be a nonempty subset of $X$ and $\Gamma: \Phi \rightarrow 2^{\boxplus}$ a multivalued map with nonempty values. Suppose the following:

(H1) $0 \geq \psi(x, y) \geq \tau(x)$ for all $x \in \Phi$ and all $y \in \Gamma(x)$,

(H2) for any $x \in \Phi$ and $\varepsilon>0$, there exists $y=y(x, \varepsilon) \in \Gamma(x)$ such that $\tau(z) \geq-\varepsilon$ for all $z \in \Gamma(y)$.

Then there exists a sizing-up function $\mu: 2^{\boxplus} \rightarrow[0, \infty]$ such that $\Gamma$ is of type $(\mu)$.

Proof. Define $\mu_{(\psi, \Gamma)}: 2^{\boxplus} \rightarrow[0, \infty]$ by

$$
\mu_{(\psi, \Gamma)}(A)= \begin{cases}0, & \text { if } A=\emptyset \\ \sup \{-\psi(x, y): x \in A, y \in \Gamma(x)\}, & \text { if } A \neq \emptyset\end{cases}
$$

Then $\mu_{(\psi, \Gamma)}$ is a sizing-up function. We will claim that $\Gamma$ is of type $\left(\mu_{(\psi, \Gamma)}\right)$. Let $x \in \mathbb{D}$ and $\varepsilon>0$ be given. By (H1) and (H2), there exists $y=y(x, \varepsilon) \in \Gamma(x)$ such that

$$
\begin{aligned}
\mu_{(\psi, \Gamma)}(\Gamma(y)) & =\sup \{-\psi(a, b): a \in \Gamma(y), b \in \Gamma(a)\} \\
& \leq \sup \{-\tau(a): a \in \Gamma(y)\} \\
& \leq \varepsilon
\end{aligned}
$$

Hence $\Gamma$ is of type $\left(\mu_{(\psi, \Gamma)}\right)$.

Theorem 3.2. Let $(X, \mathcal{U})$ be a uniform space, and let $\psi: X \times X \rightarrow(-\infty, \infty]$ and $\tau: X \rightarrow$ $[-\infty, \infty)$ be functions. Let $\Phi$ be a sequentially $(\mathcal{U})$-complete nonempty subset of $X$ and $\Gamma: \Phi \rightarrow 2^{\Phi}$ a multivalued map with nonempty values. Suppose that conditions (H1) and (H2) in Theorem 3.1 hold and further assume that

(H3) for each $x \in \boldsymbol{\Phi}, x \in \Gamma(x)$ and $\Gamma(x)$ is closed in $\boldsymbol{\Phi}$,

(H4) $x, y \in \Phi$ with $y \in \Gamma(x)$ implies that $\Gamma(y) \subseteq \Gamma(x)$,

(H5) for each $V \in \mathcal{U}$, there exists $\delta=\delta(V)>0$ such that $x, y \in \Phi$ with $y \in \Gamma(x)$ and $\psi(x, y)>-\delta$ implies that $(x, y) \in V$.

Then there exist a quasiorder $\lesssim$ on $\Phi$ and a sizing-up function $\mu: 2^{\Phi} \rightarrow[0, \infty]$ such that $(\Phi, \lesssim, \mu)$ is a $\mu$-bounded quasiordered set.

Proof. Put a binary relation $\lesssim_{(\Gamma)}$ on $\Phi$ by

$$
x \lesssim_{(\Gamma)} y \Longleftrightarrow y \in \Gamma(x)
$$

and let $S_{(\Gamma)}: \Phi \rightarrow 2^{\nexists}$ be defined by $S_{(\Gamma)}(x)=\left\{y \in \Phi: x \lesssim_{(\Gamma)} y\right\}$. Clearly, $S_{(\Gamma)}(x)=\Gamma(x)$ for each $x \in \Phi$ and $\lesssim_{(\Gamma)}$ is a quasiorder from (H3) and (H4). Let $\mu_{(\psi, \Gamma)}: 2^{\boxplus} \rightarrow[0, \infty]$ be the same as in Theorem 3.1. From the proof of Theorem 3.1, we know that $\mu_{(\psi, \Gamma)}$ is a sizing-up function and $S_{(\Gamma)}$ is of type $\left(\mu_{(\psi, \Gamma)}\right)$. We want to show that $\left(\Phi_{,} \lesssim_{(\Gamma)}, \mu_{(\psi, \Gamma)}\right)$ is a $\mu_{(\psi, \Gamma)}$-bounded 
quasiordered set. Let $c_{1} \lesssim_{(\Gamma)} c_{2} \lesssim_{(\Gamma)} \cdots \lesssim_{(\Gamma)} c_{n} \lesssim_{(\Gamma)} c_{n+1} \lesssim_{(\Gamma)} \cdots$ be a nondecreasing sequence in $\boldsymbol{D}$ satisfying

$$
\begin{aligned}
0 & =\lim _{n \rightarrow \infty} \mu_{(\psi, \Gamma)}\left(\left\{c_{n}, c_{n+1}, \cdots\right\}\right) \\
& =\lim _{n \rightarrow \infty} \sup \left\{-\psi(x, y): x \in\left\{c_{n}, c_{n+1}, \cdots\right\}, y \in S_{(\Gamma)}(x)\right\} .
\end{aligned}
$$

Since $c_{m} \in S_{(\Gamma)}\left(c_{n}\right)$ for $m, n \in \mathbb{N}$ with $m \geq n, \lim _{n, m \rightarrow \infty} \psi\left(c_{n}, c_{m}\right)=0$. Let $V \in \mathcal{U}$ and choose $W \in \mathcal{U}$ such that $W \circ W^{-1} \subset V$. By (H5), there exists $\delta=\delta(W)>0$ such that $x, y \in \mathcal{D}$ with $y \in \Gamma(x)$ and $\psi(x, y)>-\delta$ imply that $(x, y) \in W$. Since $\lim _{n, m \rightarrow \infty} \psi\left(c_{n}, c_{m}\right)=0$, there exists $n_{0} \in \mathbb{N}$ such that $\psi\left(c_{n}, c_{m}\right)>-\delta$ for all $m, n \in \mathbb{N}$ with $m \geq n \geq n_{0}$. It implies that $\left(c_{n}, c_{m}\right) \in W$ and hence $\left(c_{m}, c_{n}\right) \in W^{-1}$ for all $m, n \in \mathbb{N}$ with $m \geq n \geq n_{0}$. Since $W \circ W^{-1} \subset V$, we have $\left(c_{n}, c_{m}\right) \in V$ and $\left(c_{m}, c_{n}\right) \in V$ for $m \geq n \geq n_{0}$. Therefore, $\left\{c_{n}\right\}_{n \in \mathbb{N}}$ is a nondecreasing $(\mathcal{U})$ Cauchy sequence in $\boldsymbol{\Phi}$. By the sequential $(\mathfrak{U})$-completeness of $\boldsymbol{\Theta}$, there exists $\xi \in \boldsymbol{\Phi}$ such that $c_{n} \rightarrow \xi$ as $n \rightarrow \infty$. For each $n \in \mathbb{N}$, since $S_{(\Gamma)}\left(c_{n}\right)$ is closed from (H3) and

$$
c_{m} \in S_{(\Gamma)}\left(c_{m}\right) \subseteq S_{(\Gamma)}\left(c_{n}\right) \quad \forall m \geq n
$$

we obtain $\xi \in S_{(\Gamma)}\left(c_{n}\right)$ or $c_{n} \lesssim_{(\Gamma)} \xi$. Hence $\xi$ is an upper bound of $\left\{c_{n}\right\}$. Therefore $\left(\Phi_{,} \lesssim_{(\Gamma)}, \mu_{(\psi, \Gamma)}\right)$ is a $\mu_{(\psi, \Gamma)}$-bounded quasiordered set.

Theorem 3.3. Let $(X, \mathcal{U})$ be a Hausdorff uniform space, and let $\psi: X \times X \rightarrow(-\infty, \infty]$ and $\tau: X \rightarrow$ $[-\infty, \infty)$ be functions. Let $\Phi$ be a sequentially $(\mathcal{U})$-complete nonempty subset of $X, g: \Phi \rightarrow \Phi a$ map, and $\Gamma: \Phi \rightarrow 2^{\Phi}$ a multivalued map with nonempty values. Let $I$ be any index set. For each $i \in I$, let $F_{i}$ be a fuzzy map on $\boldsymbol{\Phi}$. Suppose the conditions (H1), (H2), (H3), and (H5) in Theorem 3.2 hold and further assume

$(\mathrm{H} 4)_{S} x, y \in \Phi$ with $y \in \Gamma(x)$ implies that $g(y) \in \Gamma(x)$ and $\Gamma(y) \subseteq \Gamma(x)$;

(H6) for any $(i, x) \in I \times \mathbb{\Phi}$, there exists $y_{(i, x)} \in \Gamma(x)$ such that $F_{i}\left(x, y_{(i, x)}\right)=1$.

Then there exists $z_{0} \in \Phi$ such that

(a) $F_{i}\left(z_{0}, z_{0}\right)=1$ for all $i \in I$,

(b) $\Gamma\left(z_{0}\right)=\left\{g\left(z_{0}\right)\right\}=\left\{z_{0}\right\}$.

Proof. Applying Theorem 3.1 and Theorem $3.2, \Gamma \equiv S_{(\Gamma)}$ is of type $\left(\mu_{(\psi, \Gamma)}\right)$ and $\left(\boldsymbol{\Phi}_{,} \lesssim_{(\Gamma)}, \mu_{(\psi, \Gamma)}\right)$ is a $\mu_{(\psi, \Gamma)}$-bounded quasiordered set, where $\lesssim_{(\Gamma)}, S_{(\Gamma)}$, and $\mu_{(\psi, \Gamma)}$ are the same as in Theorems 3.1 and 3.2. By Theorem LD, for each $x \in \mathcal{\Phi}$, there exists $v_{x} \in \Gamma(x)$ such that $\mu_{(\psi, \Gamma)}\left(\Gamma\left(v_{x}\right)\right)=0$. Then it follows from the definition of $\mu_{(\psi, \Gamma)}, v_{x} \in \Gamma\left(v_{x}\right)$, and $\mu_{(\psi, \Gamma)}\left(\Gamma\left(v_{x}\right)\right)=0$ that $\psi\left(v_{x}, z\right)=0$ for all $z \in \Gamma\left(v_{x}\right)$. We want to prove that $\Gamma\left(v_{x}\right)=\left\{v_{x}\right\}$. Since $\psi\left(v_{x}, z\right)>-\delta$ for all $z \in \Gamma\left(v_{x}\right)$ and all $\delta>0$, by (H5), we have $\left(v_{x}, z\right) \in V$ for all $z \in \Gamma\left(v_{x}\right)$ and all $V \in \mathcal{U}$. Since $\mathcal{U}$ is a Hausdorff uniformity,

$$
\left(v_{x}, z\right) \in \bigcap_{V \in \mathcal{U}} V=\Delta \quad \forall z \in \Gamma\left(v_{x}\right),
$$

and hence we have $\Gamma\left(v_{x}\right)=\left\{v_{x}\right\}$. For each $i \in I$, by $(\mathrm{H} 6), F_{i}\left(v_{x}, v_{x}\right)=1$. On the other hand, by $(\mathrm{H} 4)_{S}$, we have $g\left(v_{x}\right) \in \Gamma\left(v_{x}\right)=\left\{v_{x}\right\}$. Therefore $\Gamma\left(v_{x}\right)=\left\{g\left(v_{x}\right)\right\}=\left\{v_{x}\right\}$. The proof is completed. 
Theorem 3.4. Let $(X, \mathcal{U}), \Phi, g, \Gamma, \psi$, and $\tau$ be the same as in Theorem 3.3. Assume that the conditions (H1), (H2), (H3), (H4) $)_{S}$ and (H5) in Theorem 3.3 hold. Let I be any index set. For each $i \in I$, let $T_{i}: \Phi \rightarrow 2^{\mathbb{\Phi}}$ be a multivalued map with nonempty values. Suppose that, for each $(i, x) \in I \times \boldsymbol{\Phi}$, there exists $y_{(i, x)} \in T_{i}(x) \cap \Gamma(x)$. Then there exists $z_{0} \in \Phi$ such that

(a) $z_{0}$ is a common fixed point for the family $\left\{T_{i}\right\}_{i \in I}$ (i.e., $z_{0} \in T_{i}\left(z_{0}\right)$ for all $i \in I$ );

(b) $\Gamma\left(z_{0}\right)=\left\{g\left(z_{0}\right)\right\}=\left\{z_{0}\right\}$.

Proof. For each $i \in I$, define a fuzzy map $F_{i}$ on $\Phi$ by

$$
F_{i}(x, y)=X_{T_{i}(x)}(y)
$$

where $X_{A}$ is the characteristic function for an arbitrary set $A \subset X$. Note that $y \in T_{i}(x) \Leftrightarrow$ $F_{i}(x, y)=1$ for $i \in I$. Then for any $(i, x) \in I \times \Phi$, there exists $y_{x} \in \Gamma(x)$ such that $F_{i}\left(x, y_{x}\right)=1$. So (H6) in Theorem 3.3 holds and hence all conditions in Theorem 3.3 are satisfied. Therefore the result follows from Theorem 3.3.

Remark 3.5. Let $(X, d)$ be a complete metric space. For each $\epsilon>0$, let

$$
V(\epsilon)=\{(x, y) \in X \times X: d(x, y)<\epsilon\}
$$

It is easy to see that the family $\mathfrak{U}_{d}:=\{V(\epsilon): \epsilon>0\}$ is a Hausdorff uniformity on $X$ and $X$ is $\left(\mathcal{U}_{d}\right)$-complete.

Lemma 3.6. Let $(X, d)$ be a metric space, $g: X \rightarrow X$ a map and $\Gamma: X \rightarrow 2^{X}$ a multivalued map with nonempty values. Suppose that

(h1) for each $x \in X, x \in \Gamma(x)$,

(h2) $x, y \in X$ with $y \in \Gamma(x)$ implies that $g(y) \in \Gamma(x)$ and $\Gamma(y) \subseteq \Gamma(x)$,

(h3) if a sequence $\left\{x_{n}\right\}$ in $X$ satisfies $g\left(x_{n+1}\right) \in \Gamma\left(x_{n}\right)$ for each $n \in \mathbb{N}$, then $\lim _{n \rightarrow \infty} d\left(x_{n}, x_{n+1}\right)=0$.

Then there exist functions $\psi: X \times X \rightarrow(-\infty, \infty]$ and $\tau: X \rightarrow[-\infty, \infty)$ such that the conditions (H1) and (H2) in Theorem 3.1 hold.

Proof. Define $\psi: X \times X \rightarrow(-\infty, \infty]$ and $\tau: X \rightarrow[-\infty, \infty)$ by

$$
\begin{gathered}
\psi(x, y)=-d(x, y), \\
\tau(x)=\inf _{y \in \Gamma(x)}[-d(x, y)] .
\end{gathered}
$$

Then (H1) in Theorem 3.1 holds with $\Phi \equiv X$.

Let us verify (H2). Let $x \in X$ and $\varepsilon>0$ be given. Then there exists $\alpha \in \mathbb{N}$ such that

$$
2^{-\alpha}<\frac{\varepsilon}{4}
$$


Note first that $\tau(u)>-\infty$ for some $u \in \Gamma(x)$. Indeed, on the contrary, suppose that $\tau(z)=-\infty$ for all $z \in \Gamma(x)$. Take $z_{1} \in \Gamma(x)$. Thus $\tau\left(z_{1}\right)=\inf _{y \in \Gamma\left(z_{1}\right)}\left[-d\left(z_{1}, y\right)\right]<-1$. Hence there exists $z_{2} \in \Gamma\left(z_{1}\right)$ such that $d\left(z_{1}, z_{2}\right)>1$. Since $\tau\left(z_{2}\right)<-2$, there exists $z_{3} \in \Gamma\left(z_{2}\right)$ such that $d\left(z_{2}, z_{3}\right)>$ 2. Continuing in the process, we can obtain a sequence $\left\{z_{n}\right\} \subset X$ such that, for each $n \in \mathbb{N}$,

$$
\begin{aligned}
& \text { (i) } z_{n+1} \in \Gamma\left(z_{n}\right), \\
& \text { (ii) } d\left(z_{n}, z_{n+1}\right)>n \text {. }
\end{aligned}
$$

So, we have $\lim _{n \rightarrow \infty} d\left(z_{n}, z_{n+1}\right)=\infty$ which contradicts condition (h3). Therefore there exists $u \in \Gamma(x)$ such that $\tau(u)>-\infty$. Let $v_{1}=u$. Choose $v_{2} \in \Gamma\left(v_{1}\right)=\Gamma(u) \subseteq \Gamma(x)$ such that

$$
-d\left(v_{1}, v_{2}\right) \leq \tau\left(v_{1}\right)+\frac{1}{2}
$$

Let $k \in \mathbb{N}$ and assume that $v_{k} \in X$ is already known. Then, by induction, we obtain a sequence $\left\{v_{n}\right\}$ in $X$ such that $v_{n+1} \in \Gamma\left(v_{n}\right)$ and

$$
-d\left(v_{n}, v_{n+1}\right) \leq \tau\left(v_{n}\right)+\frac{1}{2^{n}}, \quad \text { for each } n \in \mathbb{N} .
$$

It follows that

$$
v_{n+1} \in \Gamma\left(v_{n}\right) \subseteq \Gamma\left(v_{n-1}\right) \subseteq \cdots \subseteq \Gamma\left(v_{1}\right)=\Gamma(u) \subseteq \Gamma(x), \quad \text { for each } n \in \mathbb{N}
$$

By (h2) and (h3), we have $\lim _{n \rightarrow \infty} d\left(v_{n}, v_{n+1}\right)=0$. So there exists $\beta \in \mathbb{N}$ such that

$$
d\left(v_{n}, v_{n+1}\right)<\frac{\varepsilon}{8}, \quad \forall n \in \mathbb{N} \text { with } n \geq \beta
$$

Since $\Gamma\left(v_{n+1}\right) \subseteq \Gamma\left(v_{n}\right)$ for each $n \in \mathbb{N}$, we have

$$
\begin{aligned}
\tau\left(v_{n}\right) & =\inf _{y \in \Gamma\left(v_{n}\right)}\left[-d\left(v_{n}, y\right)\right] \\
& \leq \inf _{y \in \Gamma\left(v_{n+1}\right)}\left[-d\left(v_{n+1}, y\right)+d\left(v_{n+1}, v_{n}\right)\right] \\
& =\tau\left(v_{n+1}\right)+d\left(v_{n}, v_{n+1}\right) .
\end{aligned}
$$

From (3.12) and (3.15), we obtain

$$
-\tau\left(v_{n+1}\right) \leq 2 d\left(v_{n}, v_{n+1}\right)+\frac{1}{2^{n}}, \quad n \in \mathbb{N} .
$$

Let $\gamma=\max \{\alpha, \beta\}$. Hence, combining (3.10), (3.14), and (3.16), we have

$$
0 \leq-\tau\left(v_{n+1}\right)<\frac{\varepsilon}{2}, \quad \forall n \in \mathbb{N} \text { with } n \geq \gamma
$$


Let $y_{x}=v_{\gamma+1}$. Thus, by (3.13) and (3.17), $y_{x} \in \Gamma(x)$ and $0 \leq-\tau\left(y_{x}\right)<\varepsilon / 2$. On the other hand, from the definition of $\tau$, we have

$$
0 \leq d\left(y_{x}, a\right) \leq-\tau\left(y_{x}\right), \quad \forall a \in \Gamma\left(y_{x}\right)
$$

Finally, in order to complete the proof, we need to show that $\tau(z) \geq-\varepsilon$ for all $z \in \Gamma\left(y_{x}\right)$. Let $z \in \Gamma\left(y_{x}\right)$. Then $\Gamma(z) \subseteq \Gamma\left(y_{x}\right)$ and $d\left(y_{x}, z\right) \leq-\tau\left(y_{x}\right)$. For any $w \in \Gamma(z)$, since $\Gamma(z) \subseteq \Gamma\left(y_{x}\right)$, we get

$$
d(z, w) \leq d\left(z, y_{x}\right)+d\left(y_{x}, w\right) \leq-2 \tau\left(y_{x}\right)<\varepsilon
$$

and hence it implies that $\tau(z) \geq-\varepsilon$. Therefore (H2) can be satisfied.

Theorem 3.7. Let $(X, d)$ be a complete metric space, $g: X \rightarrow X$ a map, and $\Gamma: X \rightarrow 2^{X} a$ multivalued map with nonempty values. Let $I$ be any index set. For each $i \in I$, let $F_{i}$ be a fuzzy map on X. Suppose that conditions (h2) and (h3) in Theorem 3.4 hold and further assume

(h1) for each $x \in X, x \in \Gamma(x)$ and $\Gamma(x)$ is closed,

(h4) for any $(i, x) \in I \times X$, there exists $y_{(i, x)} \in \Gamma(x)$ such that $F_{i}\left(x, y_{(i, x)}\right)=1$.

Then there exists $z_{0} \in X$ such that

(a) $F_{i}\left(z_{0}, z_{0}\right)=1$ for all $i \in I$,

(b) $\Gamma\left(z_{0}\right)=\left\{g\left(z_{0}\right)\right\}=\left\{z_{0}\right\}$.

Proof. For each $\epsilon>0$, define

$$
\begin{gathered}
V(\epsilon)=\{(x, y) \in X \times X: d(x, y)<\epsilon\}, \\
\mathcal{U}_{d}=\{V(\epsilon): \epsilon>0\} .
\end{gathered}
$$

Then $\boldsymbol{U}_{d}$ is a Hausdorff uniformity on $X$ and $X$ is $\left(\boldsymbol{U}_{d}\right)$-complete. Clearly, conditions (H3), $(\mathrm{H} 4)_{S}$, and (H6) in Theorem 3.3 hold. By Lemma 3.6, (H1) and (H2) in Theorem 3.1 holds. Let $V(\epsilon) \in \mathfrak{U}_{d}$ for $\epsilon>0$. Take $\delta(V(\epsilon)):=\epsilon>0$. If $x, y \in X$ with $y \in \Gamma(x)$ and $\psi(x, y)>-\delta(V(\epsilon))$, then $d(x, y)<\epsilon$ which means that $(x, y) \in V(\epsilon)$. So (H5) in Theorem 3.2 holds. Therefore the conclusion follows from Theorem 3.3.

Theorem 3.8. Let $(X, d), g, I$, and $\Gamma$ be the same as in Theorem 3.7. Assume that the conditions $(h 1)_{S},(h 2)$ and $(h 3)$ in Theorem 3.7 hold. Let $I$ be any index set. For each $i \in I$, let $T_{i}: X \rightarrow 2^{X}$ be a multivalued map with nonempty values. Suppose that for each $(i, x) \in I \times X$, there exists $y_{x} \in$ $T_{i}(x) \cap \Gamma(x)$. Then there exists $z_{0} \in X$ such that

(a) $z_{0}$ is a common fixed point for the family $\left\{T_{i}\right\}_{i \in I}$

(b) $\Gamma\left(z_{0}\right)=\left\{g\left(z_{0}\right)\right\}=\left\{z_{0}\right\}$.

Remark 3.9. Theorems 3.3-3.8 all generalize and improve the primitive Dancš-HegedüsMedvegyev's principle. 


\section{Some Applications to Nonlinear Problems}

The following result is a generalization of Ekeland's variational principle and Takahashi's nonconvex minimization theorem for $\tau$-functions with common fuzzy fixed point theorem.

Theorem 4.1. Let $(X, d)$ be a complete metric space, $f: X \rightarrow(-\infty, \infty]$ a proper l.s.c. and bounded from below function, $\varphi:(-\infty, \infty] \rightarrow(0, \infty)$ a nondecreasing function, and $p$ a $\tau$-function on $X$ with $p(x, \cdot)$ being l.s.c. for each $x \in X$. Let $I$ be any index set. For each $i \in I$, let $F_{i}$ be a fuzzy map on $X$. Suppose that, for each $(i, x) \in I \times X$ and any $\varepsilon>0$, there exists $y_{(i, x, \varepsilon)} \in X$ such that $F_{i}\left(x, y_{(i, x, \varepsilon)}\right)=1$ and $\varepsilon p\left(x, y_{(i, x, \varepsilon)}\right) \leq \varphi(f(x))\left(f(x)-f\left(y_{(i, x, \varepsilon)}\right)\right)$. Then for each $\varepsilon>0$ and any $u \in X$ with $f(u)<\infty$ and $p(u, u)=0$, there exists $v \in X$ such that

(a) $\varepsilon p(u, v) \leq \varphi(f(u))(f(u)-f(v))$,

(b) $\varepsilon p(v, x)>\varphi(f(v))(f(v)-f(x))$ for all $x \in X$ with $x \neq v$,

(c) $F_{i}(v, v)=1$ for all $i \in I$.

Moreover, if one further assumes that

(H) for each $\varepsilon>0$ and any $x \in X$ with $f(x)>\inf _{z \in X} f(z)$, there exists $y \in X$ with $y \neq x$ such that $\varepsilon p(x, y) \leq \varphi(f(x))(f(x)-f(y))$,

then $f(v)=\inf _{z \in X} f(z)$.

Proof. Take $g \equiv i d$ as an identity map. Let $\varepsilon>0$ be given and let $u \in X$ with $f(u)<\infty$ and $p(u, u)=0$. Put

$$
\mathcal{W}=\{x \in X: \varepsilon p(u, x) \leq \varphi(f(u))(f(u)-f(x))\} .
$$

By the lower semicontinuity of $f$ and $p(u, \cdot), \mathcal{W}$ is a nonempty closed set in $X$. So $(\mathcal{W}, d)$ is a complete metric space. Define $\Gamma: \mathcal{W} \rightarrow 2^{\mathcal{W}}$ by

$$
\Gamma(x)=\{y \in \mathcal{W}: x=y \text { or } \varepsilon p(x, y) \leq \varphi(f(x))(f(x)-f(y))\} .
$$

Then for each $x \in \mathcal{W}$, we have $x \in \Gamma(x)$ and $\Gamma(x)$ is closed. It is easy to see that if $x, y \in \mathcal{W}$ with $y \in \Gamma(x)$, then $\Gamma(y) \subseteq \Gamma(x)$. By our hypothesis, for each $(i, x) \in I \times \mathcal{W}$, there exists $y_{(i, x)} \in \Gamma(x)$ such that $F_{i}\left(x, y_{(i, x)}\right)=1$.

We will prove that if a sequence $\left\{x_{n}\right\}$ in $\mathcal{W}$ satisfies $x_{n+1} \in \Gamma\left(x_{n}\right)$ for each $n \in \mathbb{N}$, then $\lim _{n \rightarrow \infty} d\left(x_{n}, x_{n+1}\right)=0$. Let $\left\{x_{n}\right\} \subset \mathcal{W}$ satisfy $x_{n+1} \in \Gamma\left(x_{n}\right)$ for each $n \in \mathbb{N}$. Then $\left\{f\left(x_{n}\right)\right\}$ is a nonincreasing sequence. Since $f$ is bounded below, $r \equiv \lim _{n \rightarrow \infty} f\left(x_{n}\right)=\inf _{n \in \mathbb{N}} f\left(x_{n}\right)$ exists. We claim that $\lim _{n \rightarrow \infty} \sup \left\{p\left(x_{n}, x_{m}\right): m>n\right\}=0$. Let $\alpha_{n}=(1 / \varepsilon) \varphi\left(f\left(x_{1}\right)\right)\left(f\left(x_{n}\right)-r\right), n \in \mathbb{N}$. For $m, n \in \mathbb{N}$ with $m>n$, since $\varphi$ is nondecreasing, we have

$$
p\left(x_{n}, x_{m}\right) \leq \sum_{j=n}^{m-1} p\left(x_{j}, x_{j+1}\right) \leq \alpha_{n}
$$

Then $\sup \left\{p\left(x_{n}, x_{m}\right): m>n\right\} \leq \alpha_{n}$ for each $n \in \mathbb{N}$. Since $\lim _{n \rightarrow \infty} f\left(x_{n}\right)=r$, we obtain $\lim _{n \rightarrow \infty} \alpha_{n}=0$ and $\lim _{n \rightarrow \infty} \sup \left\{p\left(x_{n}, x_{m}\right): m>n\right\}=0$. By Lemma 2.1, $\left\{x_{n}\right\}$ is a 
Cauchy sequence in $\mathcal{W}$, and hence we have $\lim _{n \rightarrow \infty} d\left(x_{n}, x_{n+1}\right)=0$. So all the conditions of Theorem 3.7 are satisfied. Applying Theorem 3.7, there exists $v \in \mathcal{W}$ such that

$$
\begin{gathered}
F_{i}(v, v)=1, \quad \forall i \in I, \\
\Gamma(v)=\{v\} .
\end{gathered}
$$

Since $v \in \mathcal{W}$, we have the conclusion (a). From (4.5), $\varepsilon p(v, x)>\varphi(f(v))(f(v)-f(x))$ for all $x \in \mathcal{W}$ with $x \neq v$. For any $x \in X \backslash \mathcal{W}$, since

$$
\begin{aligned}
\varepsilon[p(u, v)+p(v, x)] & \geq \varepsilon p(u, x) \\
& >\varphi(f(u))(f(u)-f(x)) \\
& \geq \varepsilon p(u, v)+\varphi(f(v))(f(v)-f(x)),
\end{aligned}
$$

it follows that $\varepsilon p(v, x)>\varphi(f(v))(f(v)-f(x))$ for all $x \in X \backslash \mathcal{W}$. So the conclusion (b) holds.

Moreover, assume that condition (H) holds. On the contrary, if $f(v)>\inf _{x \in X} f(x)$, then there exists $w \in X$ with $w \neq v$ such that $\varepsilon p(v, w) \leq \varphi(f(v))(f(v)-f(w))$. But, by (b), we have

$$
\varepsilon p(v, w)>\varphi(f(v))(f(v)-f(w)) \geq \varepsilon p(v, w)
$$

a contradiction. Therefore $f(v)=\inf _{z \in X} f(z)$. The proof is completed.

By using Theorem 4.1, we can immediately obtain the following $\tau$-function version of generalized Ekeland's variational principle, generalized Takahashi's nonconvex minimization theorem, and generalized Caristi's common fixed point theorem for multivalued maps.

Theorem 4.2. Let $(X, d), f, \varphi$, and $p$ be the same as in Theorem 4.1. Let I be any index set. For each $i \in I$, let $T_{i}: X \rightarrow 2^{X}$ be a multivalued map with nonempty values such that, for each $(i, x) \in I \times X$ and any $\varepsilon>0$, there exists $y_{(i, x)} \in T_{i}(x)$ such that $\varepsilon p\left(x, y_{(i, x)}\right) \leq \varphi(f(x))\left(f(x)-f\left(y_{(i, x)}\right)\right)$. Then for each $\varepsilon>0$ and $u \in X$ with $f(u)<\infty$ and $p(u, u)=0$, there exists $v \in X$ such that

(a) $\varepsilon p(u, v) \leq \varphi(f(u))(f(u)-f(v))$,

(b) $\varepsilon p(v, x)>\varphi(f(v))(f(v)-f(x))$ for all $x \in X$ with $x \neq v$,

(c) $v$ is a common fixed point for the family $\left\{T_{i}\right\}_{i \in I}$.

Moreover, if one further assumes that

(H) for each $\varepsilon>0$ and any $x \in X$ with $f(x)>\inf _{z \in X} f(z)$, there exists $y \in X$ with $y \neq x$ such that $\varepsilon p(x, y) \leq \varphi(f(x))(f(x)-f(y))$,

then $f(v)=\inf _{z \in X} f(z)$.

Remark 4.3. Theorem 4.2 extends some results in $[2,8,14,15,19,22]$ and references therein.

The following result is an existence theorem of nonconvex version of variational disclusion problem with common fuzzy fixed point theorem in metric spaces. 
Theorem 4.4. Let $(X, d)$ be a complete metric space, E a nonempty set with $\alpha \in E$, and $L: X \times X \rightarrow$ $2^{E}$ a multivalued map. Let $I$ be any index set. For each $i \in I$, let $F_{i}$ be a fuzzy map on X. Assume that

(D1) for each $x \in X$, the set $\{y \in X: x=y$ or $\alpha \in L(x, y)\}$ is a closed subset of $X$,

(D2) $x, y, z \in X$ with $\alpha \in L(x, y)$ and $\alpha \in L(y, z)$ implies that $\alpha \in L(x, z)$,

(D3) if a sequence $\left\{x_{n}\right\}_{n \in \mathbb{N}}$ in $X$ satisfies $\alpha \in L\left(x_{n}, x_{n+1}\right)$ for each $n \in \mathbb{N}$, then $d\left(x_{n}, x_{n+1}\right) \rightarrow 0$ as $n \rightarrow \infty$,

(D4) for any $(i, x) \in I \times X$, there exists $y_{(i, x)} \in X$ such that $\alpha \in L\left(x, y_{(i, x)}\right)$ and $F_{i}\left(x, y_{(i, x)}\right)=1$.

Then there exists $v \in X$ such that

(a) $F_{i}(v, v)=1$ for all $i \in I$,

(b) $\alpha \notin L(v, x)$ for all $x \in X \backslash\{v\}$.

Proof. Take $g \equiv i d$ as an identity map. Define $\Gamma: X \rightarrow 2^{X}$ by

$$
\Gamma(x)=\{y \in X: x=y \text { or } \alpha \in L(x, y)\} .
$$

Clearly, (h1) ${ }_{S}$ (h3), and (h4) in Theorem 3.7 hold. To see (h2), let $x, y \in X$ with $y \in$ $\Gamma(x)$. We need to consider the following two possible cases:

Case 1. If $x=y$, then $\Gamma(y)=\Gamma(x) \subseteq \Gamma(x)$ is obvious.

Case 2. If $x \neq y$, then $\alpha \in L(x, y)$. For any $z \in \Gamma(y)$, if $z=y$, one has $z \in \Gamma(x)$. Otherwise, if $\alpha \in L(y, z)$, then it follows from $\alpha \in L(x, y)$ and (D2) that $\alpha \in L(x, z)$. So $z \in \Gamma(x)$. Therefore $\Gamma(y) \subseteq \Gamma(x)$. such that

By Cases 1 and 2, we prove that (h2) holds. Applying Theorem 3.7, there exists $v \in X$

(1) $F_{i}(v, v)=1$ for all $i \in I$,

(2) $\Gamma(v)=\{v\}$.

From (2), we obtain $\alpha \notin L(v, x)$ for all $x \in X \backslash\{v\}$.

Remark 4.5. Theorem 4.4 generalizes [17, Theorems 3.1] which is one of the main results of Lin and Chuang [17].

Here, we give an example illustrating Theorem 4.4.

Example 4.6. Let $X=[0,3]$ with the metric $d(x, y)=|x-y|$ for $x, y \in X$. Then $(X, d)$ is a complete metric space. Let $E=(-1000,-500) \cup\{-360\} \cup\{-210\} \cup[-100, \infty)$ and let $L$ : $X \times X \rightarrow 2^{E}$ be defined by

$$
L(x, y)=[9(x-y)+5, \infty)
$$


Let $C_{0}=\{0\}$ and $C_{n}=(n-1, n]$, for every $n \in\{1,2,3\}$, and define a fuzzy map $F: X \times X \rightarrow$ $[0,1]$ by

$$
F(x, y)= \begin{cases}0, & \text { if }(x, y) \in C_{0} \times C_{0} \\ \frac{1}{4}, & \text { if }(x, y) \in X \times C_{1} \\ \frac{1}{2}, & \text { if }(x, y) \in X \times C_{2} \\ 1, & \text { if }(x, y) \in X \times C_{3}\end{cases}
$$

Clearly, $5 \in L(x, x)$ for each $x \in X$. Note that, for each $x \in X,\{y \in X: x=y$ or $5 \in L(x, y)\}=$ $\{y \in X: 5 \in L(x, y)\}=[x, 3]$ is nonempty and closed in X. So (D1) and (D4) hold. To see (D2), let $x, y, z \in X$ with $5 \in L(x, y)$ and $5 \in L(y, z)$. It is easy to see that $5 \in L(x, z)$ holds. Finally, let $\left\{x_{n}\right\}_{n \in \mathbb{N}}$ be a sequence in $X$ satisfing $5 \in L\left(x_{n}, x_{n+1}\right)$ for each $n \in \mathbb{N}$. So $\left\{x_{n}\right\}$ is a nondecreasing sequence and $x_{n} \leq 3$ for each $n \in \mathbb{N}$. Thus $\left\{x_{n}\right\}$ converges in $X$ and hence $d\left(x_{n}, x_{n+1}\right) \rightarrow 0$ as $n \rightarrow \infty$. So (D3) also holds. By Theorem 4.4, there exists $v \in X$ (in fact, we take $v=3)$ such that $F(v, v)=1$ and $5 \notin L(v, y)$ for all $y \in X \backslash\{v\}$.

The following conclusion is immediate from Theorem 4.4.

Theorem 4.7. Let $(X, d), E, \alpha, L,(D 1),(D 2)$, and (D3) be the same as in Theorem 4.4. Let I be any index set. For each $i \in I$, let $T_{i}: X \rightarrow 2^{X}$ be a multivalued map with nonempty values. Suppose that for each $(i, x) \in I \times X$, there exists $y_{(i, x)} \in T_{i}(x)$ such that $\alpha \in L\left(x, y_{(i, x)}\right)$.

Then there exists $v \in X$ such that

(a) $v$ is a common fixed point for the family $\left\{T_{i}\right\}_{i \in I}$,

(b) $\alpha \notin L(v, x)$ for all $x \in X \backslash\{v\}$.

Following a similar argument as in Theorem 4.4, we can easily obtain the following existence theorem of nonconvex version of variational inclusion problem in metric spaces.

Theorem 4.8. In Theorem 4.4, if conditions (D1) and (D2) are replaced by the condition (D1)' and $(D 2)^{\prime}$, where

(D1)' for each $x \in X$, the set $\{y \in X: x=y$ or $\alpha \notin L(x, y)\}$ is a closed subset of $X$,

$(D 2)^{\prime} x, y, z \in X$ with $\alpha \notin L(x, y)$ and $\alpha \notin L(y, z)$ implies that $\alpha \notin L(x, z)$,

then there exists $v \in X$ such that

(a) $F_{i}(v, v)=1$ for all $i \in I$,

(b) $\alpha \in L(v, x)$ for all $x \in X \backslash\{v\}$.

Theorem 4.9. Let $(X, d), E, \alpha, L,(D 1)^{\prime},(D 2)^{\prime}$, and $(D 3)$ be the same as in Theorem 4.8. Let I be any index set. For each $i \in I$, let $T_{i}: X \rightarrow 2^{X}$ be a multivalued map with nonempty values. Suppose that for each $(i, x) \in I \times X$, there exists $y_{(i, x)} \in T_{i}(x)$ such that $\alpha \in L\left(x, y_{(i, x)}\right)$. 
Then there exists $v \in X$ such that

(a) $v$ is a common fixed point for the family $\left\{T_{i}\right\}_{i \in I}$,

(b) $\alpha \in L(v, x)$ for all $x \in X \backslash\{v\}$.

The following existence theorem of nonconvex version of variational inclusion and disclusion problem in the Ekeland's sense is immediate from Theorem 4.4.

Theorem 4.10. Let $(X, d)$ be a complete metric space, $p$ a $\tau$-function on $X$ with $p(x, \cdot)$ being l.s.c. for each $x \in X, V$ a topological vector space with origin $\theta, G: X \times X \rightarrow 2^{V}$ a multivalued maps, and $k_{0} \in V \backslash\{\theta\}$. Let I be any index set. For each $i \in I$, let $F_{i}$ be a fuzzy map on X. Assume that

(S1) for each $x \in X$, the set $\left\{y \in X: x=y\right.$ or $\left.\theta \in G(x, y)+p(x, y) k_{0}\right\}$ is closed in $X$,

(S2) $x, y, z \in X$ with $\theta \in G(x, y)+p(x, y) k_{0}$ and $\theta \in G(y, z)+p(y, z) k_{0}$ implies that $\theta \in G(x, z)+p(x, z) k_{0}$,

(S3) if a sequence $\left\{x_{n}\right\}_{n \in \mathbb{N}}$ in $X$ satisfies $\theta \in G\left(x_{n}, x_{n+1}\right)+p\left(x_{n}, x_{n+1}\right) k_{0}$ for each $n \in \mathbb{N}$, then $d\left(x_{n}, x_{n+1}\right) \rightarrow 0$ as $n \rightarrow \infty$,

(S4) for any $(i, x) \in I \times X$, there exists $y_{(i, x)} \in X$ such that $\theta \in G\left(x, y_{(i, x)}\right)+p\left(x, y_{(i, x)}\right) k_{0}$ and $F_{i}\left(x, y_{(i, x)}\right)=1$.

Then for each $u \in X$ with $\theta \in G(u, u)$ and $p(u, u)=0$, there exists $v \in X$ such that

(i) $\theta \in G(u, v)+p(u, v) k_{0}$,

(ii) $\theta \notin G(v, x)+p(v, x) k_{0}$ for all $X \backslash\{v\}$,

(iii) $F_{i}(v, v)=1$ for all $i \in I$.

Proof. Let $u \in X$ be given and $L: X \times X \rightarrow 2^{V}$ defined by $L(x, y):=G(x, y)+p(x, y) k_{0}$ for $(x, y) \in X \times X$. Put $\mathcal{M}:=\{x \in X: \theta \in L(u, x)\}$. Since $u \in \mathcal{M}, \mathcal{M} \neq \emptyset$. By (S1), $(\mathcal{M}, d)$ be a complete metric space. It is not hard to see that all conditions in Theorem 4.4 are satisfied from (S1)-(S4). Applying Theorem 4.4, there exists $v \in \mathcal{M}$ such that $F_{i}(v, v)=1$ for all $i \in I$ and $\theta \notin L(v, x)$ for all $x \in \mathcal{M} \backslash\{v\}$ or, equivalently,

(a) $\theta \in G(u, v)+p(u, v) k_{0}$,

(b) $\theta \notin G(v, x)+p(v, x) k_{0}$ for all $\mathcal{M} \backslash\{v\}$.

For any $x \in X \backslash \mathcal{M}$, if $\theta \in G(v, x)+p(v, x) k_{0}$, then, by (S2) and (a), we have $x \in \mathcal{M}$, which is a contradiction. Therefore $\theta \notin G(v, x)+p(v, x) k_{0}$ for all $X \backslash\{v\}$.

Remark 4.11. Theorem 3.2 in [17] is a special case of Theorem 4.10.

\section{Acknowledgments}

The author wishes to express his hearty thanks to the anonymous referees for their helpful suggestions and comments improving the original draft. This research was supported by the National Science Council of Taiwan. 


\section{References}

[1] S. Danč̌, M. Hegedús, and P. Medvegyev, "A general ordering and fixed-point principle in complete metric space," Acta Scientiarum Mathematicarum, vol. 46, no. 1-4, pp. 381-388, 1983.

[2] D. H. Hyers, G. Isac, and T. M. Rassias, Topics in Nonlinear Analysis and Applications, World Scientific, River Edge, NJ, USA, 1997.

[3] L.-J. Lin and W.-S. Du, "From an abstract maximal element principle to optimization problems, stationary point theorems and common fixed point theorems," Journal of Global Optimization, vol. 46, no. 2, pp. 261-271, 2010.

[4] E. Bishop and R. R. Phelps, "The support functionals of a convex set," in Proc. Sympos. Pure Math., Vol. VII, pp. 27-35, American Mathematical Society, Providence, RI, USA, 1963.

[5] H. Brézis and F. E. Browder, "A general principle on ordered sets in nonlinear functional analysis," Advances in Mathematics, vol. 21, no. 3, pp. 355-364, 1976.

[6] A. Brøndsted, "On a lemma of Bishop and Phelps," Pacific Journal of Mathematics, vol. 55, pp. 335-341, 1974.

[7] W.-S. Du, "On some nonlinear problems induced by an abstract maximal element principle," Journal of Mathematical Analysis and Applications, vol. 347, no. 2, pp. 391-399, 2008.

[8] B. G. Kang and S. Park, "On generalized ordering principles in nonlinear analysis," Nonlinear Analysis: Theory, Methods E Applications, vol. 14, no. 2, pp. 159-165, 1990.

[9] L.-J. Lin and W.-S. Du, "On maximal element theorems, variants of Ekeland's variational principle and their applications," Nonlinear Analysis: Theory, Methods E Applications, vol. 68, no. 5, pp. 12461262, 2008.

[10] G. X.-Z. Yuan, KKM Theory and Applications in Nonlinear Analysis, vol. 218 of Monographs and Textbooks in Pure and Applied Mathematics, Marcel Dekker, New York, NY, USA, 1999.

[11] M. Amemiya and W. Takahashi, "Fixed point theorems for fuzzy mappings in complete metric spaces," Fuzzy Sets and Systems, vol. 125, no. 2, pp. 253-260, 2002.

[12] S. S. Chang and Q. Luo, "Caristi's fixed point theorem for fuzzy mappings and Ekeland's variational principle," Fuzzy Sets and Systems, vol. 64, no. 1, pp. 119-125, 1994.

[13] A. Göpfert, C. Tammer, and C. Zălinescu, “On the vectorial Ekeland's variational principle and minimal points in product spaces," Nonlinear Analysis: Theory, Methods \& Applications, vol. 39, no. 7, pp. 909-922, 2000.

[14] A. Granas and C. D. Horvath, "On the order-theoretic Cantor theorem," Taiwanese Journal of Mathematics, vol. 4, no. 2, pp. 203-213, 2000.

[15] O. Kada, T. Suzuki, and W. Takahashi, "Nonconvex minimization theorems and fixed point theorems in complete metric spaces," Mathematica Japonica, vol. 44, no. 2, pp. 381-391, 1996.

[16] G. M. Lee, B. S. Lee, J. S. Jung, and S. S. Chang, "Minimization theorems and fixed point theorems in generating spaces of quasi-metric family," Fuzzy Sets and Systems, vol. 101, no. 1, pp. 143-152, 1999.

[17] L.-J. Lin and C.-S. Chuang, "Existence theorems for variational inclusion problems and the set-valued vector Ekeland variational principle in a complete metric space," Nonlinear Analysis: Theory, Methods E Applications, vol. 70, no. 7, pp. 2665-2672, 2009.

[18] L.-J. Lin and W.-S. Du, "Ekeland's variational principle, minimax theorems and existence of nonconvex equilibria in complete metric spaces," Journal of Mathematical Analysis and Applications, vol. 323, no. 1, pp. 360-370, 2006.

[19] L.-J. Lin and W.-S. Du, "Some equivalent formulations of the generalized Ekeland's variational principle and their applications," Nonlinear Analysis: Theory, Methods \& Applications, vol. 67, no. 1, pp. 187-199, 2007.

[20] L.-J. Lin and W.-S. Du, "Systems of equilibrium problems with applications to new variants of Ekeland's variational principle, fixed point theorems and parametric optimization problems," Journal of Global Optimization, vol. 40, no. 4, pp. 663-677, 2008.

[21] T. Suzuki, "Generalized distance and existence theorems in complete metric spaces," Journal of Mathematical Analysis and Applications, vol. 253, no. 2, pp. 440-458, 2001.

[22] W. Takahashi, Nonlinear Functional Analysis, Fixed Point Theory and Its Applications, Yokohama Publishers, Yokohama, Japan, 2000.

[23] Chr. Tammer, "A generalization of Ekeland's variational principle," Optimization, vol. 25, no. 2-3, pp. 129-141, 1992.

[24] R. K. Bose and D. Sahani, "Fuzzy mappings and fixed point theorems," Fuzzy Sets and Systems, vol. 21, no. 1, pp. 53-58, 1987. 
[25] S. S. Chang, "Fixed point theorems for fuzzy mappings," Fuzzy Sets and Systems, vol. 17, no. 2, pp. 181-187, 1985.

[26] S. S. Chang and Y. G. Zhu, "On variational inequalities for fuzzy mappings," Fuzzy Sets and Systems, vol. 32, no. 3, pp. 359-367, 1989.

[27] G. Moţ and A. Petruşel, "Fixed point theory for a new type of contractive multivalued operators," Nonlinear Analysis: Theory, Methods E Applications, vol. 70, no. 9, pp. 3371-3377, 2009.

[28] G. Petruşel, "Existence and data dependence of fixed points and strict fixed points for multivalued Y-contractions," Carpathian Journal of Mathematics, vol. 23, no. 1-2, pp. 172-176, 2007.

[29] I. A. Rus, "Strict fixed point theory," Fixed Point Theory, vol. 4, no. 2, pp. 177-183, 2003.

[30] L. Guran, "Fixed points for multivalued operators with respect to a $w$-distance on metric spaces," Carpathian Journal of Mathematics, vol. 23, no. 1-2, pp. 89-92, 2007.

[31] L. Guran and A. Petruşel, "Existence and data dependence for multivalued weakly Cirić-contractive operators," Acta Universitatis Sapientiae. Mathematica, vol. 1, no. 2, pp. 151-159, 2009. 